TRANSACTIONS OF THE

AMERICAN MATHEMATICAL SOCIETY

Volume 295, Number 2, June 1986

\title{
DEFINABLE SETS IN ORDERED STRUCTURES. II
}

\author{
JULIA F. KNIGHT ${ }^{1}$, ANAND PILLAY ${ }^{2}$ AND CHARLES STEINHORN ${ }^{3}$
}

\begin{abstract}
It is proved that any 0-minimal structure $M$ (in which the underlying order is dense) is strongly 0 -minimal (namely, every $N$ elementarily equivalent to $M$ is 0 -minimal). It is simultaneously proved that if $M$ is 0 minimal, then every definable set of $n$-tuples of $M$ has finitely many "definably connected components."
\end{abstract}

0. Introduction. In this paper we study the structure of definable sets (of tuples) in an arbitrary 0-minimal structure $M$ (in which the underlying order is dense).

Recall from [PS1, PS2] that the structure $M$ is said to be 0-minimal if $M=$ $\left(M,<, R_{i}\right)_{i \in I}$, where < is a total ordering on $M$ and every definable (with parameters) subset of $M$ is a finite union of points in $M$ and intervals $(a, b)$ where $a \in M$ or $a=-\infty$ and $b \in M$ or $b=+\infty$.

$M$ is said to be strongly 0 -minimal if every $N$ which is elementarily equivalent to $M$ is 0-minimal. We will always assume that the underlying order of $M$ is a dense order with no first or least element.

In this paper we also introduce the notion of a definable set $X \subset M^{n}$ being definably connected, and we prove

THEOREM 0.1. Let $M$ be 0-minimal. Then any definable $X \subset M^{n}$ is a disjoint union of finitely many definably connected definable sets.

THEOREM 0.2. If $M$ is 0-minimal, then $M$ is strongly 0-minimal.

THEOREM 0.3. (a) Let $M$ be 0-minimal and let $\phi\left(x_{1}, \ldots, x_{n}, y_{1}, \ldots, y_{m}\right)$ be any formula of $L$ (the language for $M$ ). Then there is $K<\omega$ such that for any $\bar{b} \in M^{m}$, the set $\phi(\bar{x}, \bar{b})^{M}\left(=\left\{\bar{a} \in M^{n}: M \vDash \phi(\bar{a}, \bar{b})\right\}\right)$ has at most $K$ definably connected components.

(b) If $M$ is a $\mathbf{0 - m i n i m a l ~ e x p a n s i o n ~ o f ~}(\mathbf{R},<)$, then in (a) we can replace definably connected by connected.

Theorems 0.1 and 0.2 are proved simultaneously by a rather complicated induction argument (outlined in $\S 3$ and undertaken in $\S \S 4$ and 5 ). Theorem 0.3 follows from Theorems 0.1 and 0.2 by a compactness argument.

Let us remark that if $M$ is the field of real numbers, or more generally any real closed field, then by Tarski's quantifier elimination $[\mathbf{T}], M$ is (strongly) 0 -minimal and moreover the definable sets (of $n$-tuples) in $M$ are precisely the semialgebraic

Received by the editors April 15, 1985.

1980 Mathematics Subject Classification. Primary 03C45.

Key words and phrases. 0-minimal, definably connected, cell.

1 Partially supported by NSF grant MCS 83-01272-01.

${ }^{2}$ Partially supported by NSF grant DMS 84-01713.

${ }^{3}$ Partially supported by NSF grant DMS 84-03137. 
sets. Thus Theorem $0.3(\mathrm{~b})$ implies results on the (topological) character of semialgebraic sets in $\mathbf{R}^{n}$ due to Whitney $[\mathbf{W}]$ and Mather $[\mathbf{M}]$.

What is striking is that we prove these results in a general model-theoretic context and under general (model-theoretic) assumptions, in particular abstracting from any algebraic or analytic structure. The possibility of such proofs was first envisaged by van den Dries, whose paper $[\mathbf{D}]$ inspired both the general definition of 0 -minimality and the work in this paper.

In fact in $[\mathbf{D}]$, van den Dries proved Theorem 0.1 under the stronger assumptions that $M$ is strongly 0 -minimal and the underlying order on $M$ is that of $\mathbf{R}$. Van den Dries' proof involves the notion of cylindrical algebraic decomposition introduced by Collins $[\mathbf{C}]$ to give a fast quantifier elimination for the real field. Our proof (of Theorem 0.1) (insofar as we drop van den Dries' assumption that $M \uparrow\{<\} \simeq$ $(\mathbf{R},<)$ ) follows in outline that of van den Dries.

Finally we should say something concerning the attribution of the results among the authors.

In [PS1] the following (formally) weaker version of Theorem 0.1 was announced:

THEOREM 0.1'. Let $M$ be strongly 0-minimal. Then any definable set $X \subset M^{n}$ is a disjoint union of finitely many definably connected definable sets.

Similarly we announced Theorem 0.3 with 0 -minimal replaced by strongly 0 minimal.

Originally these weaker results were to have appeared in [PS2]. However, it was decided instead to incorporate them into the present article, as the proof of Theorem 0.2 needed the characterization of definable sets of higher dimensions. Thus, that part of the proof of Theorem 0.1 which depends on the assumption of strong 0-minimality (essentially the inductive proofs of 3.5 and 3.6) is due in outline to Pillay and Steinhorm. The rest (essentially the proof of 3.7) and thus the proof of Theorem 0.2 modulo (the proof of) Theorem $0.1^{\prime}$ is due to Knight and Pillay.

The various component parts of the proof were put together, and the paper written, by Pillay. Pillay would also like to thank Mati Rubin for some helpful discussions in the summer of 1983.

1. Preliminaries. $M$ will, throughout this paper, denote an arbitrary but fixed 0 -minimal structure whose underlying order $<$ is dense with no first or last element. We work throughout in $M$ and thus we will be rather lax with our model-theoretic terminology. By a definable set, we mean an $X \subset M^{n}$ (some $n \geq 1$ ) such that for some (first order) formula $\phi\left(x_{1}, \ldots, x_{n}\right) \in L(M)(L(M)$ being the language $L$ of $M$ together with names for $M), X=\left\{\bar{a} \in M^{n}: M \vDash \phi(\bar{a})\right\}$.

We will often write just $\phi(\bar{a})$ in place of the more formal $M \vDash \phi(\bar{a})$. Also we often leave to the reader the (usually routine) verification that certain sets we describe are definable.

$M$ comes equipped with a topology, namely the interval topology, a basic open set being one of the form $(a, b)(=\{x: a<x<b\})$ (where $a, b \in M \cup\{ \pm \infty\})$. (We will try to distinguish the case when $(a, b)$ denotes an interval in $M$ and when it denotes a point in $M^{2}$.) For any $n>1, M^{n}$ is given the product topology. By an open box in $M^{n}$ we mean a set of the form $X_{1} \times X_{2} \times \cdots \times X_{n}$ where each $X_{i}$ is an interval $\left(a_{i}, b_{i}\right)$ in $M$. So the basic open sets in $M^{n}$ are the open boxes. 
Similarly we can define a closed box in $M^{n}$. Clearly any such box (open or closed) is definable.

Thus the following makes sense:

DEFinition 1.1. Let $X, Y$ be subsets of $M^{n}$ with $Y \subset X$. We say that $\bar{a} \in M^{n}$ is a boundary point of $Y$ in $X$ if $\bar{a} \in X$ and for every open box $B \subset M^{n}$ containing $\bar{a}, B \cap X$ intersects both $Y$ and the complement of $Y$. If $X=M^{n}$ we just say $\bar{a}$ is a boundary point of $Y$.

Let us look at the special case where $n=1, X$ is an interval $(a, b)$ of $M$ and $Y$ is a definable subset of $X$. By 0 -minimality $Y$ has a finite number of boundary points in $X$, say $a_{0}<a_{1}<\cdots<a_{m}$. Note that there are six possible kinds of such boundary point $a_{i}$ :

kind 1: $a_{i} \notin Y, a_{i}$ is the right endpoint of some interval in $Y$, and the left endpoint of some interval in $Y$, 1 ,

kind 2: $a_{i} \notin Y, a_{i}$ is the right endpoint of some interval in $Y$ and is not of kind

kind 3: $a_{i} \notin Y, a_{i}$ is the left endpoint of some interval in $Y$ and is not of kind 1, kind 4: $a_{i} \in Y$ and $a_{i}$ is the right endpoint of some interval in $Y$,

kind 5: $a_{i} \in Y$ and $a_{i}$ is the left endpoint of some interval in $Y$,

kind 6: $a_{i}$ is an isolated point of $Y$.

In this context we say

DEFINITION 1.2. The type of $Y$ in $X$ is the sequence $\left\langle\operatorname{kind}\left(a_{0}\right), \ldots, \operatorname{kind}\left(a_{m}\right)\right\rangle$ where $a_{0}<a_{1}<\cdots<a_{m}$ are the boundary points of $Y$ in $X$.

Let $X$ be an interval $(a, b)$ in $M$. By an isomorphism on $X$ we mean a map $f: X \rightarrow M$ such that $f$ is either an order preserving or order reversing map from $X$ onto some interval $(c, d)$. (Again, $a, b, c, d \in M \cup\{ \pm \infty\}$.)

One of the main technical results of [PS2] and a cornerstone of this paper is the following

Proposition 1.3. Let $X=(a, b)$ be an interval in $M$, and $f$ a definable function from $X$ to $M$. Then there are points $a=a_{0}<a_{1}<\cdots<a_{n}=b$ in $M$ such that, on each interval $\left(a_{i}, a_{i+1}\right), f$ is either constant or an isomorphism. Moreover, if $X$ and $f$ are $A$-definable, then the $a_{i}$ can be taken to be A-definable.

A trivial but important consequence is

COROLLARY 1.4. Let $f$ be a definable function from $(a, b)$ into $M$. Then both $\lim _{x \rightarrow a^{+}} f(x)$ and $\lim _{x \rightarrow b^{-}} f(x)$ exist in $M \cup\{ \pm \infty\}$.

Proof. By 1.3 there is $b^{\prime}$ with $a<b^{\prime}<b$ such that $f \uparrow\left(b^{\prime}, b\right)$ is either constant or an isomorphism. If $f \uparrow\left(b^{\prime}, b\right)$ is constant with value $c$, then clearly $\lim _{x \rightarrow b^{-}} f(x)=c$. If $f \uparrow\left(b^{\prime}, b\right)$ is an isomorphism of $\left(b^{\prime}, b\right)$ with $(c, d)$ (where of course maybe $c=-\infty$ or $d=+\infty)$, then $\lim _{x \rightarrow b^{-}} f(x)=d$ if $f$ is order preserving and $\lim _{x \rightarrow b^{-}} f(x)=c$ if $f$ is order reversing. Similarly for $\lim _{x \rightarrow a^{+}} f(x)$.

Now we introduce some "nice" definable sets (of $M^{n}$ ) which for the purpose of this paper we call cells. These cells as well as their dimension are defined inductively:

Definition 1.5. (i) If $X=\{a\}$, where $a \in M$, then $X$ is a cell and $\operatorname{dim} X=0$. If $X$ is an interval $(a, b),(a, b \in M \cup \pm \infty)$, then $X$ is a cell and $\operatorname{dim} X=1$.

(ii) Suppose that $X \subset M^{n}$ is a cell with $\operatorname{dim} X=k$. We define two kinds of cells in $M^{n+1}$ associated with $X$ : 
(a) Let $f: X \rightarrow M$ be definable and continuous. Then

$$
X_{1}=\operatorname{graph}(f) \underset{\mathrm{df}}{=}\{(\bar{x}, f(\bar{x})): \bar{x} \in X\}
$$

is a cell in $M^{n+1}$ and we put $\operatorname{dim} X_{1}=k$.

(b) Let $f_{1}, f_{2}$ be definable continuous functions from $X$ to $M \cup\{ \pm \infty\}$ with $f_{1}<f_{2}$ on $X$. Then

$$
X_{2}=\left(f_{1}, f_{2}\right)_{X} \underset{\mathrm{df}}{=}\left\{(\bar{x}, y): \bar{x} \in X, f_{1}(\bar{x})<y<f_{2}(\bar{x})\right\}
$$

is a cell in $M^{n+1}$ and we put $\operatorname{dim} X_{2}=k+1$.

(iii) Nothing else is a cell.

REMARK 1.6. Any cell is definable. If $X \subset M^{n}$ is a cell, then $\operatorname{dim} X \leq n$, and moreover $\operatorname{dim} X=n$ iff $X$ is open (in $M^{n}$ ). For any cell $X, \operatorname{dim} X=0$ iff $X$ is a point.

ProOF. Easy, by induction.

Now for $X$ a cell in $M^{n}$ with $n>1$ and $\operatorname{dim} X<n$, we construct (as in [D]) a definable homeomorphism $h_{X}$ of $X$ with a cell $X^{\prime}$ in $M^{n-1}$.

If $X=\operatorname{graph}(f)$, where $f$ is continuous on the cell $Y$ in $M^{n-1}$, we let $h_{X}: X \rightarrow Y$ be the projection map. If $X=(f, g)_{Y}$ (as in Definition 1.5(ii)(b)), then clearly $Y$ is a cell in $M^{n-1}$ with $\operatorname{dim} Y<n-1$, and we have two cases;

Case (i): $n-1>1$. Then by induction we have some $H_{Y}: Y \rightarrow Y^{\prime}\left(Y^{\prime}\right.$ a cell in $\left.M^{n-2}\right)$ and we put $X^{\prime}=\left(f h_{Y}^{-1}, g h_{Y}^{-1}\right)_{Y^{\prime}}$, and $h_{X}(\bar{x}, y)=\left(h_{Y}(\bar{x}), y\right)$.

Case (ii): $n-1=1$. Thus $\operatorname{dim} Y=0$ and $Y$ is a point $a \in M$. In this case we put $X^{\prime}=(f(a), g(a))$ in $M$ and $h_{X}(a, y)=y$.

Clearly in both Case (i) and Case (ii) $X^{\prime}$ is a cell and $h_{X}$ a definable homeomorphism of $X$ with $X^{\prime}$.

Let us remark that if $X$ is a cell in $M^{n}$ with $\operatorname{dim} X>0$, then by composing functions $h$ described above we see that $X$ is definably homeomorphic to an open cell $Y$ in $M^{k}$, where $k=\operatorname{dim} X$.

2. Definable connectedness. In this section we introduce the notion of definable connectedness and we show that cells are definably connected. (It is worth noting that what we shall call a "definably connected definable set" corresponds exactly to what Brumfiel [B] calls a "connected semialgebraic set" in the case of an arbitrary real closed field.)

Let $Y \subset X$ be subsets of $M^{n}$. As usual we say that $Y$ is open in $X$ if there is some open $Y^{\prime} \subset M^{n}$ such that $Y^{\prime} \cap X=Y$. The following fact (which we include for completeness) says that if in addition $X, Y$ are definable, then $Y^{\prime}$ can also be chosen definable.

Proposition 2.1. Let $Y, X$ be definable subsets of $M^{n}$ with $Y \subset X$. Then the following are equivalent.

(i) $Y$ is open in $X$,

(ii) for each $\bar{a} \in Y$ there is some open box $B$ in $M^{n}$ containing $\bar{a}$ such that $B \cap X \subset Y$,

(iii) there is an open, definable $Y^{\prime} \subset M^{n}$ such that $Y^{\prime} \cap X=Y$.

Proof. (i) $\rightarrow$ (ii) is clear, as is (iii) $\rightarrow$ (i).

(ii) $\rightarrow$ (iii): Assume (ii) and let $Y^{\prime}=\bigcup\left\{B: B\right.$ is an open box in $M^{n}$ such that $\bar{a} \in B$ for some $\bar{a} \in Y$ and $B \cap X \subset Y\}$. Clearly $Y^{\prime}$ is open and also definable. If 
$\bar{a} \in Y$, then by (ii) $\bar{a} \in Y$, so $Y \subset Y^{\prime}$. Also by the definition of $Y^{\prime}, Y^{\prime} \cap X \subset Y$. Thus $Y^{\prime} \cap X=Y$ and we have proved (iii).

DEFINITION 2.2. Let $X \subset M^{n}$ be definable. We say that $X$ is definably connected if there do not exist disjoint definable sets $Y_{1}, Y_{2}$ both open in $X$ such that $X=$ $Y_{1} \cup Y_{2}$.

The following is then easy (see Definition 1.1):

LEMMA 2.3. Let $X \subset M^{n}$ be definable. Then $X$ is definably connected if and only if whenever $Y \varsubsetneqq X$ is definable, then $X$ contains some boundary point of $Y$ in $X$.

Proposition 2.4. Let $X \subset M^{n}$ be a cell. Then $X$ is definably connected.

PROOF. The proof is by induction on $n$. If $n=1$, then $X$ is clearly definably connected by the definition of 0 -minimality. So suppose $n>1$ and $X$ is a cell in $M^{n}$. If $\operatorname{dim} X=0$ then $X$ is a point, so clearly definably connected. We have two further cases:

Case (i): $\operatorname{dim} X=k<n(k>0)$. Then $h_{X}(X)$ is a cell in $M^{n-1}$ (where $h_{X}$ is the definable homeomorphism defined in $\S 1)$. By induction hypothesis, $h_{X}(X)$ is definably connected, and this easily lifts up to $X$.

Case (ii): $\operatorname{dim} X=n$ (i.e. $X$ is open). Thus $X=(f, g)_{Z}$, where $Z$ must be an open cell in $M^{n-1}$. Now let $Y \varsubsetneqq X$ be definable. We want to show that $X$ contains a boundary point of $Y$ in $X$ (and use Lemma 2.3). Let $Z^{\prime}=\{\bar{a} \in Z: \exists b(\bar{a}, b) \in$ $Y \wedge \exists b(\bar{a}, b) \in X-Y\}$.

Case (ii) $)_{\mathrm{a}}: Z^{\prime}$ is nonempty. Fix $\bar{a} \in Z^{\prime}$ and let $T_{\bar{a}}=\{b:(\bar{a}, b) \in Y\}$. So $Y_{\bar{a}}$ is a definable subset of the interval $(f(\bar{a}), g(\bar{a}))$, and so (by the case $n=1),(f(\bar{a}), g(\bar{a}))$ contains some $b$ which is a boundary point for $Y_{\bar{a}}$ in $(f(\bar{a}), g(\bar{a}))$. Clearly $(\bar{a}, b)$ is then a boundary point for $Y$ in $X$.

Case (ii) $: Z^{\prime}$ is empty. Let $Z_{1}=\{\bar{a} \in Z: \forall b[(a, b) \in X \rightarrow(a, b) \in Y]\}$, and $Z_{2}=\{\bar{a} \in Z: \forall b[(\bar{a}, b) \in X \rightarrow(\bar{a}, b) \notin Y]\}$. Thus $Z=Z_{1} \dot{\cup} Z_{2}$ and $Z_{1}, Z_{2}$ are both nonempty. By induction hypothesis, $Z$ contains a boundary point $\bar{a}$ for $Z_{1}$ in $Z$. Let $b$ be such that the point $(\bar{a}, b) \in X$. We claim that $(\bar{a}, b)$ is a boundary point of $Y$ in $X$.

So let $B$ be a box in $M^{n}$ containing $(\bar{a}, b)$. We may assume $B \subset X$ (as $X$ is open). Let $B_{1}=\pi(B)$, the projection of $B$ onto the first $n-1$ coordinates. So $B_{1} \subset Z$, and $\bar{a} \in B_{1}$. Thus (as $\bar{a}$ is a boundary point of $Z_{1}$ in $Z$ ), there are $\bar{a}_{1} \in B_{1} \cap Z_{1}, \bar{a}_{2} \in B_{1} \cap Z_{2}$. Let $b_{1}, b_{2}$ be such that $\left(\bar{a}_{1}, b_{1}\right),\left(\bar{a}_{2}, b_{2}\right) \in B$. Clearly $\left(\bar{a}_{1}, b_{1}\right) \in Y,\left(\bar{a}_{2}, b_{2}\right) \in X-Y$. So $(\bar{a}, b)$ is a boundary point of $Y$ in $X$, completing the proof of the proposition.

We conclude this section with a definable version of the "Bolzano Intermediate Value Theorem" (which we do not actually use).

Proposition 2.5. Let $X \subset M^{n}$ be definable and definably connected. Let $f: X \rightarrow M$ be definable and continuous. Let $\bar{a}_{1}, \bar{a}_{2} \in X$ and $b \in M$ be such that $f\left(\bar{a}_{1}\right)<b<f\left(\bar{a}_{2}\right)$. Then for some $\bar{a} \in X, f(\bar{a})=b$.

PROOF. Left to the reader.

3. Outline of the proofs. Roughly speaking, our proofs of the main theorems will go as follows: We will prove inductively, for each $n$ : (i) any definable set $X$ in 
$M^{n}$ is a finite union of cells, (ii) any definable function $f: M^{n} \rightarrow M$ is "piecewise" continuous (where the pieces are restrictions of $f$ to cells in $M^{n}$ of which there are a finite number), (iii) if $\phi(\bar{x}, y)$ is an $L(M)$ formula with $l(\bar{x})=n$ such that $\left|\phi(\bar{a}, y)^{M}\right|$ is finite for all $\bar{a} \in M^{n}$, then $\left|\phi(\bar{a}, y)^{M}\right| \leq K$ for some $K<\omega$ and all $\bar{a} \in M^{n}$.

Theorems 0.1 and 0.2 will fall out of (i), (iii) respectively, quite easily. In fact we shall prove rather finer versions of (i)-(iii) which we will give below. We need some preliminary definitions.

DEFINITION 3.1. Let $X \subset M^{n}$. Let $\phi(\bar{x}, y) \in L(M)$, where $l(\bar{x})=n$. $\phi$ is said to be finite on $X$ if $\phi(\bar{a}, y)^{M}$ is finite for all $\bar{a} \in X$, and uniformly finite on $X$ if there is $K<\omega$ such that $\left|\phi(\bar{a}, y)^{M}\right| \leq K$ for all $\bar{a} \in X$.

DEFINITION 3.2. Let $X \subset M^{n}$ be definable and let $\phi(\bar{x}, y)$ be finite on $X$. The first point function of $\phi$ on $X$ is the function $f_{1}$ assigning to each $\bar{a} \in X$ for which there is a $b$ with $\phi(\bar{a}, b)$ the least such $b$. Similarly for the last point function $f_{l}$ of $\phi$ on $X$. (These functions are clearly definable.)

Definition 3.3. Let $X \subset M^{n}$ be definable. By a decomposition $P$ of $X$ we mean a partition of $X$ into finitely many cells. If $X^{\prime} \subset X$ is definable we say that such $P$ partitions $X^{\prime}$ if $Y \subset X^{\prime}$ or $Y \cap X^{\prime}=\emptyset$ for every $Y \in P$.

DEFINITION 3.4. Let $X$ be an open cell in $M^{n}$. Let $\phi(\bar{x}, y) \in L(M)$ be finite on $X$. The point $\bar{a} \in X$ is said to be good for $\phi(\bar{x}, y)$ if

(i) $\forall b[\phi(\bar{a}, b) \rightarrow$ there is an open box $B \subset X$ containing $\bar{a}$ and an open interval $I$ containing $b$ such that $\phi(x, y)^{M} \cap(B \times I)$ is the graph of a continuous function from $B$ to $I]$, and

(ii) $\forall b\left[\neg \phi(\bar{a}, b) \rightarrow\right.$ there is an open box in $M^{n+1}$ containing $(\bar{a}, b)$ such that $\left.\phi(\bar{x}, y)^{M} \cap B=\emptyset\right]$.

If $a \in X$ is not good for $\phi, \bar{a}$ is said to be bad for $\phi$.

In $\S \S 4$ and 5 we will prove the following three statements inductively for all $N<\omega:$

$(3.5)_{N}$ Let $X$ be a cell in $M^{N}$ and let $X_{i}, i \in I$, be a finite collection of definable subsets of $X$. Then there is a decomposition $\mathcal{P}$ of $X$ which partitions each $X_{i}$.

$(3.6)_{N}$ Let $X \subset M^{N}$ be a cell and $f: X \rightarrow M$ a definable function. Then there is a decomposition $P$ of $X$ such that for each $Y \in \mathcal{P}, f \uparrow Y$ is continuous.

$(3.7)_{N}$ (a) Let $X \subset M^{N}$ be a cell and suppose that $\phi(\bar{x}, y) \in L(M)(l(\bar{x})=N)$ is finite on $X$. Then $\phi(\bar{x}, y)$ is uniformly finite on $X$.

(b) Let $X \subset M^{N}$ be an open cell. Suppose that $\phi(\bar{x}, y) \in L(M)$ is finite on $X$ and that the first and last point functions of $\phi$ on $X$ are continuous. Suppose also that each $\bar{a} \in X$ is good for $\phi$. Then for all $\bar{a}_{1}, \bar{a}_{2} \in X$

$$
\left|\phi\left(\bar{a}_{1}, y\right)^{M}\right|=\left|\phi\left(\bar{a}_{2}, y\right)^{M}\right| \text {. }
$$

4. The case $N=1$. Here we prove statements $(3.5)-(3.7)$ for $N=1$.

Now for $N=1,(3.5)$ is an immediate consequence of the 0-minimality of $M$, a cell in $M$ being an open interval or a point. Also (3.6) follows from Proposition 1.3 .

So we are left with (3.7) $(N=1)$ which is actually where the hard work in this paper is. We first prove $(3.7)(\mathrm{b})$.

ProOF OF (3.7)(b) FOR $N=1$. Let $X=(a, b)$ be an interval in $M$, and suppose that $\phi(x, y) \in L(M)$ is finite on $X$ and that the first and last point functions of $\phi$ 
on $X$ are continuous. Assume also that every $c \in X$ is good for $\phi$. We will show that $\left|\phi\left(c_{1}, y\right)^{M}\right|=\left|\phi\left(c_{2}, y\right)^{M}\right|$ for all $c_{1}, c_{2} \in X$. Suppose not and we will get a contradiction. So for some $k<\omega, X_{1}=\left\{c \in X:\left|\phi(c, y)^{M}\right|=k\right\}$ is a nonempty proper subset of $X$.

Let (by 0-minimality) $c \in X$ be a boundary point for $X_{1}$ in $X$. Let $\phi(c, y)^{M}=$ $\left\{d_{1}, \ldots, d_{L}\right\}$, where $d_{1}<d_{2}<\cdots<d_{L}$. As $c$ is good for $\phi$ it follows that there are an interval $I \subset X$ containing $c$ and disjoint intervals $J_{1}, \ldots, J_{L}$ containing $d_{1}, \ldots, d_{L}$ respectively such that $\phi(x, y) \cap\left(I \times J_{i}\right)$ is the graph of a continuous function from $I \rightarrow J_{i}$ for each $i=1, \ldots, L$. For each $i=1, \ldots, L$ let $g_{i}$ denote the function $I \rightarrow J$ of which $\phi(x, y) \cap\left(I \times J_{1}\right)$ is the graph. Note that each $g_{i}$ is definable and $g_{1}<g_{2}<\cdots<g_{L}$ on $I$. Thus we have

$$
\left|\phi\left(c^{\prime}, y\right)^{M}\right| \geq L \text { for all } c^{\prime} \in I \text {. }
$$

Now, as $c$ is a boundary point of $X_{1}$ in $X$, it follows from (*) that for some interval $I^{\prime} \subset X$ to the left or right of $c$ (i.e. $I^{\prime}=(d, c)$ or $(c, d)$ ),

$$
\left|\phi\left(c^{\prime}, y\right)^{M}\right|>L \quad \text { for all } c^{\prime} \in I^{\prime} \text {. }
$$

Without loss of generality $I^{\prime}=(c, d)(d>c)$, and $I^{\prime} \subset I$.

We define the following function $g$ on $I^{\prime}$ : For $c^{\prime} \in I^{\prime}, g\left(c^{\prime}\right)=$ least $d^{\prime}$ such that $\phi\left(c^{\prime}, d^{\prime}\right)$ and $d^{\prime} \neq g_{i}\left(c^{\prime}\right)$ for all $i=1, \ldots, L$.

Note that $g$ is definable and moreover by $(* *)$, the definition of the $g_{i}$ and the fact that $\phi\left(c^{\prime}, y\right)^{M}$ is finite for all $c^{\prime} \in I^{\prime}, g$ is defined on all of $I^{\prime}$.

Let us also remark that $f_{1}\left(c^{\prime}\right)<g\left(c^{\prime}\right)<f_{l}\left(c^{\prime}\right)$ for all $c^{\prime} \in I$, where $f_{1}, f_{l}$ are the first and last point functions of $\phi$ on $X$. Now by Corollary 1.4 (and the fact that $f_{1}, f_{l}$ are continuous on $\left.X\right)$ it follows that $\lim _{x \rightarrow c^{+}} g(x)$ exists and is not $\pm \infty$. So let $d=\lim _{x \rightarrow c^{+}} g(x)$.

Now we have either

Case (i): $\phi(c, d)$. In this case $d=d_{i}$ for some $i=1, \ldots, L$. Then $d=$ $\lim _{x \rightarrow c^{+}} g(x)=\lim _{x \rightarrow c^{+}} g_{i}(x)$, and for all $c^{\prime} \in I^{\prime}, g\left(c^{\prime}\right) \neq g_{i}\left(c^{\prime}\right)$ and both $\phi\left(c^{\prime}, g\left(c^{\prime}\right)\right)$, $\phi\left(c^{\prime}, g_{i}\left(c^{\prime}\right)\right)$. This clearly contradicts clause (i) (Definition 3.4 of $c$ being good for $\phi)$; or

Case (ii): $\neg \phi(c, d)$. In this case clearly clause (ii) of Definition 3.4 is contradicted for $c$.

This contradiction proves that $\left|\phi\left(c_{1}, y\right)^{M}\right|=\left|\phi\left(c_{2}, y\right)^{M}\right|$ for all $c_{1}, c_{2} \in X$.

PROOF OF (3.7)(a) FOR $N=1$. Let $\phi(x, y) \in L(M)$ be finite on the cell $X$ in $M$. We want to show that $\phi$ is uniformly finite on $X$. If $X$ is a singleton, this is clear. So we may assume that $X$ is an interval $(a, b)$. We may (by 0 -minimality or more precisely $\left.(3.5)_{1}\right)$ assume also that for all $c \in(a, b)$ there is $y$ with $\phi(c, y)$. Thus the first and last point functions $f_{1}, f_{l}$ of $\phi$ are defined on $X$. By $(3.6)_{1}$ we may assume also that both $f_{1}$ and $f_{l}$ are continuous on $X$.

Let $Y=\{c \in(a, b): c$ is bad for $\phi\}$.

Case (i): $Y$ is finite, say $Y=\left\{a_{1}, \ldots, a_{m}\right\}$ with $a<a_{1}<\cdots<a_{m}<b$. Thus on each of the intervals $\left(a, a_{1}\right),\left(a_{1}, a_{2}\right), \ldots,\left(a_{m}, b\right), f_{1}$ and $f_{l}$ are continuous and every point is good for $\phi$. By (3.7)(b) $(N=1)$ above, $\phi$ is uniformly finite on $(a, b)$.

Case (ii): $Y$ is infinite. We now seek a contradiction!

By 0 -minimality, $Y$ (a definable set) contains an infinite interval which again we call $(a, b)$. Thus every $c \in(a, b)$ is bad for $\phi$. Now $c$ being bad for $\phi$ is, by Definition 
3.4, witnessed by some $d$ such that, either

(I) $\phi(c, d)$, and for all intervals $I$ around $c$ and $J$ around $d, \phi(x, y)^{M} \cap(I \times J)$ is not the graph of a continuous function from $I$ to $J$; or $\varnothing$.

(II) $\neg \phi(c, d)$, but for every box $B$ in $M^{2}$ around the point $(c, d), \phi(x, y)^{M} \cap B \neq$

In either case we call $(c, d)$ a nasty point for $\phi$; if (I) holds we call $(c, d)$ nasty of type (I), and if (II) holds we call $(c, d)$ nasty of type (II).

ClAIM 4.1 (UNDER CURRENT HYPOTHESES). For any $c \in(a, b)$ there is a least $d$ such that $(c, d)$ is nasty for $\phi$.

Proof OF Claim 4.1. Fix $c \in(a, b)$. Clearly there are only finitely many $d$ such that $(c, d)$ is nasty of type (I). So it suffices to show

(*) if for all $d$ in the interval $\left(d_{1}, d_{2}\right),(c, d)$ is nasty of type (II), then $\left(c, d_{1}\right)$ is nasty.

Now if $\neg \phi\left(c, d_{1}\right)$, then easily $\left(c, d_{1}\right)$ is nasty of type (II). On the other hand, suppose $\phi\left(c, d_{1}\right)$. Now for every box $B$ containing the point $\left(c, d_{1}\right)$, and for every $d \in\left(d_{1}, d_{2}\right)$ such that $(c, d) \in B$ there are points $\left(c^{\prime}, d^{\prime}\right)$ arbitrarily close to $(c, d)$ satisfying $\phi\left(c^{\prime}, d^{\prime}\right)$. So clearly $\left(c, d_{1}\right)$ is nasty of type (I). Thus $(*)$ and Claim 4.1 are proved.

Thus we can make

DEFINITION 4.2. For any $c \in(a, b)$, let $g(c)$ be the least $d$ such that $(c, d)$ is nasty for $\phi$.

Clearly $g$ is definable.

Now by 0 -minimality and (3.6) 1 we can find a subinterval $X^{\prime} \subset(a, b)$ such that $g$ is continuous on $X^{\prime}$ and moreover either $(c, g(c))$ is nasty of type (I) for all $c \in X^{\prime}$, or $(c, g(c))$ is nasty of type (II) for all $c \in X^{\prime}$.

Again we assume $X^{\prime}=(a, b)$.

Case (ii) $\mathrm{a}$. For all $c \in(a, b),(c, g(c))$ is nasty of type (I). Let us define the functions $g_{1}, g_{2}$ on the interval $(a, b)$ as follows:

For any $c \in(a, b)$

$g_{1}(c)=\left\{\begin{array}{l}\text { the greatest } y \text { such that } \phi(c, y) \text { and } y<g(c) \text { if there is such a } y, \\ -\infty \text { otherwise, }\end{array}\right.$

$g_{2}(c)=\left\{\begin{array}{l}\text { the smallest } y \text { such that } \phi(c, y) \text { and } y>g(c) \text { if there is such a } y, \\ +\infty \text { otherwise. }\end{array}\right.$

So $g_{1}, g_{2}$ are "definable". We can again assume (by $\left.(3.5)_{1},(3.6)_{1}\right)$ that both $g_{1}, g_{2}$ are "continuous" on $(a, b)$ (i.e. either $g_{1}$ is defined on $(a, b)$ and continuous or $g_{1}(x)=-\infty \forall x \in(a, b)$, and either $g_{2}$ is defined and continuous on $(a, b)$ or $\left.g_{2}(x)=+\infty \forall x \in(a, b)\right)$.

Now let $c \in(a, b)$.

Claim 4.3. $(c, g(c))$ is not nasty for $\phi$. (This will be a contradiction.)

PROOF OF Claim 4.3. Let $d_{1}, d_{2}$ be such that

$$
g_{1}(c)<d_{1}<g(c)<d_{2}<g_{2}(c) \text {. }
$$

Now as $g_{1}, g, g_{2}$ are continuous at $c$ (and as $g_{1}<g<g_{2}$ on $(a, b)$ ), there is some interval $I \subset(a, b)$ containing $c$ such that for all $c^{\prime} \in I$,

$$
g_{1}\left(c^{\prime}\right)<d_{1}, \quad g_{2}\left(c^{\prime}\right)>d_{2} \quad \text { and } g\left(c^{\prime}\right) \in\left(d_{1}, d_{2}\right)
$$


It follows from $(*)$, the subcase hypothesis, the definition of $g_{1}, g_{2}$ and the continuity of $g$, that $\phi(x, y)^{M} \cap\left(I \times\left(d_{1}, d_{2}\right)\right)$ is the graph of $g$, i.e., the graph of a continuous function from $I \rightarrow\left(d_{1}, d_{2}\right)$. Thus $(c, g(c))$ is not nasty, proving the claim (and giving a contradiction).

Case (ii) . For all $c \in(a, b),(c, g(c))$ is nasty of type (II).

Note that in this case, for all $c \in(a, b)$

$$
f_{1}(c)<g(c)<f_{l}(c)
$$

Again we define, for $c \in(a, b)$,

$$
\begin{aligned}
& g_{1}(c)=\text { greatest } y \text { such that } \phi(c, y) \text { and } y<g(c) \\
& g_{2}(c)=\text { least } y \text { such that } \phi(c, y) \text { and } y>g(c)
\end{aligned}
$$

Now we can assume $g_{1}, g_{2}$ to be continuous on $(a, b)$.

Again let $c \in(a, b)$ and we make

ClaIM 4.4. $(c, g(c))$ is not nasty for $\phi$.

PROOF OF CLAIM 4.4. By the continuity of $g_{1}, g_{2}$ we can again find an interval $J$ containing $g(c)$ and an interval $I$ containing $c$ such that $g_{1}\left(c^{\prime}\right), g_{2}\left(c^{\prime}\right) \notin J$ for all $c^{\prime} \in I$, and $g\left(c^{\prime}\right) \in J$ for all $c^{\prime} \in I$.

As $\phi\left(c^{\prime}, g\left(c^{\prime}\right)\right)$ for all $c^{\prime} \in I$ and by the definition of $g_{1}, g_{2}$ we see that $\phi(x, y)^{M} \cap$ $(I \times J)=\varnothing$. Thus $(c, g(c))$ is not nasty for $\phi$. (Again we have a contradiction.)

Thus we have shown that Case (ii) leads to a contradiction and this completes the proof of (3.7)(a) for $N=1$. We have proved (3.5)-(3.7) for $N=1$.

5. The case $N>1$ (the induction step). In this section let $N>1$. We first prove

Proposition 5.1. Assume $(3.5)_{n},(3.6)_{n}$ and $(3.7)_{n}$ for all $n<N$. Then $(3.5)_{N}$ holds.

ProOF. Let $X$ be a cell in $M^{N}$ and $\left\{X_{i}: i \in I\right\}$ a finite collection of definable subsets of $X$. If $\operatorname{dim} X<N$, let $h_{X}$ be the definable homeomorphism of $X$ with some cell $X^{\prime}$ in $M^{N-1}$ defined in $\S 1$. By assumption there is a decomposition $P^{\prime}$ of $X^{\prime}$ which partitions each $h_{X}\left(X_{i}\right)$. It is clear that $P^{\prime}$ lifts via $h_{X}^{-1}$ to a decomposition $P$ of $X$ which partitions each $X_{i}$.

So let us suppose that $\operatorname{dim} X=N$. Let $X^{0}=\pi(X)$ (where $\pi$ is the projection onto the first $N-1$ coordinates).

Thus $X=(f, g)_{X^{0}}$, where $f, g$ are continuous functions from $X^{0}$ to $M$, with $f<g$ on $X^{0}$.

By $(3.5)_{N-1}$ there is a decomposition $\mathcal{P}$ of $X^{0}$ which partitions each $\pi\left(X_{i}\right)$. Note that $\left\{(f, g)_{Y^{0}}: Y^{0} \in P\right\}$ is a decomposition of $X$.

Fix some open $Y^{0} \in \mathcal{P}$ and let $I^{\prime}=\left\{i \in X: \pi\left(X_{i}\right) \cap Y \neq \varnothing\right\}$. Let $Y=(f, g)_{Y^{0}}$. It is clearly enough to show

LEMMA 5.2. There is a decomposition of $Y$ which partitions each $X_{i}, i \in I^{\prime}$.

Proof of Lemma 5.2. For any $\bar{a} \in Y^{0}$ and $i \in I^{\prime}$, let $\left(X_{i}\right)_{\bar{a}}=\left\{b:(\bar{a}, b) \in X_{i}\right\}$. So $\left(X_{i}\right)_{\bar{a}}$ is always a nonempty subset of $(Y)_{\bar{a}}=(f(\bar{a}), g(\bar{a}))$. Let bd $\left(\left(X_{i}\right)_{\bar{a}}\right)=$ the set of boundary points of $\left(X_{i}\right)_{\bar{a}}$ in $(Y)_{\bar{a}}$ (for each $\bar{a} \in Y^{0}, i \in I^{\prime}$ ). By (3.7) $)_{N-1}$ (a), for each $i \in I^{\prime}, \operatorname{bd}\left(\left(X_{1}\right)_{\bar{a}}\right)$ is uniformly finite as $\bar{a}$ ranges over $Y^{0}$. 
By $(3.5)_{N-1}$ we can assume that there are, for each $i \in I^{\prime}, k_{i}<\omega$ such that

(i) for each $i \in I^{\prime}$, for all $\bar{a} \in Y^{0},\left|\operatorname{bd}\left(X_{i}\right)_{\bar{a}}\right|=k_{i}$, and

(ii) for each $i \in I^{\prime}$, for any $\bar{a}_{1}, \bar{a}_{2} \in Y^{0},\left(X_{i}\right)_{\bar{a}_{1}}$ and $\left(X_{i}\right)_{\bar{a}_{2}}$ have the same type in $(Y)_{\bar{a}_{1}},(Y)_{\bar{a}_{2}}$ respectively (see Definition 1.2 ).

Now for each $i \in I^{\prime}$ and $a \in Y^{0}$, let $f_{i}^{1}(\bar{a}), \ldots, f_{i}^{k_{i}}(\bar{a})$ be the first, $\ldots, k_{i}$ th members of bd $\left(\left(X_{i}\right)_{\bar{a}}\right)$.

Again by $(3.5)_{N-1}$ we can assume that

(iii) for any $i, j \in I^{\prime}$ and $1 \leq m \leq k_{i}, 1 \leq l \leq k_{j}$, we have

$$
\begin{aligned}
& \text { either for all } \bar{a} \in Y^{0}, f_{i}^{m}(\bar{a})=f_{j}^{l}(\bar{a}), \\
& \text { or for all } \bar{a} \in Y^{0}, f_{i}^{m}(\bar{a})<f_{j}^{l}(\bar{a}), \\
& \text { or for all } \bar{a} \in Y^{0}, f_{i}^{m}(\bar{a})>f_{j}^{l}(\bar{a}) .
\end{aligned}
$$

Thus by (iii) we can write the set of functions $\left\{f_{i}^{j}: i \in I^{\prime}, 1 \leq j \leq k_{i}\right\}$ as a sequence (without repetitions) $\left\langle g_{1}, \ldots, g_{k}\right\rangle$ such that

$$
1 \leq i<j \leq k \Rightarrow g_{i}<g_{j} \text { on } Y^{0} .
$$

Finally, several applications of $(3.6)_{N-1}$ to the $g_{i}$ allow us to assume that

(iv) each of $g_{1}, \ldots, g_{k}$ is continuous on $Y^{0}$.

Now clearly (by (iii) and (iv)) the collection of definable sets

$$
\left\{\left(f, g_{1}\right)_{Y^{0}}\right\} \cup\left\{\left(g_{i}, g_{i+1}\right)_{Y^{0}}: 1 \leq i<k\right\} \cup\left\{\left(g_{k}, g\right)_{Y^{0}}\right\} \cup\left\{\operatorname{graph}\left(g_{i}\right): i=1, \ldots, k\right\}
$$

is a decomposition of $Y$ which by (ii) above partitions each $X_{i}, i \in I^{\prime}$.

This proves Lemma 5.2, and so also Proposition 5.1.

Proposition 5.3. Assume that $(3.5)_{n}$ and $(3.6)_{n}$ hold for all $n<N$ and that $(3.5)_{N}$ holds. Then $(3.6)_{N}$ holds.

Proof. Let $X$ be a cell in $M^{N}$ and $f: X \rightarrow M$ a definable function. We must find a decomposition $P$ of $X$ such that $f \uparrow Y$ is continuous for each $Y \in P$. If $\operatorname{dim} X<N$, then $f \circ h_{X}^{-1}$ is a definable function on the cell $h_{X}(X)$ in $M^{N-1}$. (3.6) $)_{N-1}$ gives us a decomposition $P^{\prime}$ of $h_{X}(X)$ into cells on each of which $f \circ h_{X}^{-1}$ is continuous. Clearly an application of $h_{X}^{-1}$ gives us our required decomposition of $X$.

So we may assume that $X$ is open in $M^{N}$. Let us define: $X_{1}=\{(\bar{a}, b) \in$ $X: f\left(x_{1}, \ldots, x_{N-1}, b\right)$ is continuous on some box $B$ (in $M^{N-1}$ ) containing $\bar{a}$ such that $B \times\{b\} \subset X\}$ and $X_{2}=\left\{(\bar{a}, b) \in X: f\left(\bar{a}, x_{N}\right)\right.$ is either constant or an isomorphism on some open interval $I$ containing $b$ such that $\{\bar{a}\} \times I \subset X\}$.

By $(3.5)_{N}$ we obtain a decomposition $P$ of $X$ which partitions both $X_{1}$ and $X_{2}$. It is clearly enough to show

LeMma 5.4. For any open $Y \in P, f$ is continuous on $Y$.

Proof of LemMa 5.4. Pick some open $Y \in P$. Thus $Y=\left(f_{1}, f_{2}\right)_{\pi(Y)}$, where $\pi(Y)$ is the projection of $Y$ on the first $N-1$ coordiantes and $f_{1}<f_{2}$ are both continuous on $\pi(Y)$. We now make some claims.

Claim 5.5. $Y \subset X_{1}$.

PROOF. Let $(\bar{a}, b) \in Y$. So clearly the function $f\left(x_{1}, \ldots, x_{N}, b\right)$ is defined on some open subset of $\pi(Y)$. 
By (3.5) $)_{N-1}$ and (3.6) $)_{N-1}$ there is some open cell $Z$ in $M^{N-1}$ such that $Z \subset$ $\pi(Y)$ and $f(\bar{x}, b)$ is continuous on $Z$. Let $\bar{a}^{\prime} \in Z$, so clearly $\left(\bar{a}^{\prime}, b\right) \in X_{1}$. Thus as $Y \subset X_{1}$ or $Y \cap X_{1}=\varnothing$ we must have that $Y \subset X_{1}$, proving Claim 5.5.

ClaIM 5.6. $Y \subset X_{2}$ and moreover, for each $\bar{a} \in \pi(Y)$, the function $f\left(\bar{a}, x_{N}\right)$ is either constant or an isomorphism on the interval $\left(f_{1}(\bar{a}), f_{2}(\bar{a})\right)$.

PROOF. The fact that $Y \subset X_{2}$ follows by an argument as in the proof of Claim 5.5 (using Proposition 1.3). Now for the rest. Let $\bar{a} \in \pi(Y)$. By Proposition 1.3 , there are $b_{1}, \ldots, b_{m}$ with $f_{1}(a)<b_{1}<b_{2}<\cdots<b_{m}<f_{2}(\bar{a})$ such that on each subinterval $\left(f_{1}(\bar{a}), b_{1}\right),\left(b_{m}, f_{2}(\bar{a})\right)$ and $\left(b_{i}, b_{i+1}\right), f\left(\bar{a}, x_{N}\right)$ is constant or an isomorphism, and that $m$ is least possible. If $m=0$ we are finished. Otherwise we see that $f\left(\bar{a}, x_{N}\right)$ can neither be constant nor an isomorphism on any open interval $I$ containing $b_{1}$. This contradicts the fact that $Y \subset X_{2}$ (as clearly $\left.\left(\bar{a}, b_{1}\right) \in Y\right)$, and proves this claim.

We can now complete the proof of Lemma 5.4.

Let $(\bar{a}, b) \in Y$. Let $J$ be some interval containing $f(\bar{a}, b)$. We want to find an open box $B$ (in $M^{N}$ ) containing $(\bar{a}, b)$ such that $f(B) \subset J$.

First by Claim 5.6 we can find some closed interval $I$ containing $b$ (in its interior), $I \subset\left(f_{1}(\bar{a}), f_{2}(\bar{a})\right)$ such that $f\left(\bar{a}, x_{N}\right)(I) \subset J$. Let $I=\left[b_{1}, b_{2}\right]$. By Claim 5.5 we can find open boxes $B_{1}, B_{2}$ in $M^{N-1}$, both containing $\bar{a}$ such that

(*) $\quad B_{1} \times\left\{b_{1}\right\} \subset Y, B_{2} \times\left\{b_{2}\right\} \subset Y$ and $f\left(\bar{x}, b_{1}\right)\left(B_{1}\right) \subset J, f\left(\bar{x}, b_{2}\right)\left(B_{2}\right) \subset J$.

Let $B^{\prime}$ be an open box in $M^{N-1}$ containing $\bar{a}$ such that $B^{\prime} \subset B_{1} \cap B_{2}$. Note that $B^{\prime} \times I \subset Y$.

We assert that

$$
f\left(B^{\prime} \times I\right) \subset J .
$$

Let $\bar{a} \in B^{\prime}, b^{\prime} \in I$. So $f_{1}\left(\bar{a}^{\prime}\right)<b_{1}<b^{\prime}<b_{2}<f_{2}\left(\bar{a}^{\prime}\right)$, and by $(*) f\left(\bar{a}^{\prime}, b_{1}\right) \in J$ and $f\left(\bar{a}^{\prime}, b_{2}\right) \in J$. By by Claim 5.6, $f\left(\bar{a}^{\prime}, x_{N+1}\right)$ is constant or an isomorphism on $\left(f_{1}\left(\bar{a}^{\prime}\right), f_{2}\left(\bar{a}^{\prime}\right)\right)$. Thus clearly $f\left(\bar{a}^{\prime}, b^{\prime}\right) \in J$. This proves $(* *)$, Lemma 5.4 and so also Proposition 5.3.

Finally we have to prove

Proposition 5.7. Assume $(3.5)_{n}$ and $(3.6)_{n}$ for all $n \leq N$, and $(3.7)_{n}$ for all $n<N$. Then $(3.7)_{N}$ holds.

ProOF. We first prove $(3.7)_{N}(\mathrm{a})$. Let $\phi\left(x_{1}, \ldots, x_{N}, y\right)$ be an $L(M)$-formula and $X$ a cell in $M^{N}$ such that $\phi(\bar{x}, y)$ is finite on $X$. We want to show that $\phi$ is uniformly finite on $X$.

If $\operatorname{dim} X<N$, then $h_{X}$ gives us a definable bijection of $X$ with a cell $X^{\prime}$ in $M^{N-1}$. Let $\psi\left(x_{1}, \ldots, x_{N-1}, y\right)$ be the formula $\phi\left(h_{X}^{-1}\left(x_{1}, \ldots, x_{N}\right), y\right)$. So $\psi\left(x_{1}, \ldots, x_{N-1}, y\right)$ is uniformly finite on $X^{\prime}$ by $(3.7)_{N-1}$ and thus $\phi\left(x_{1}, \ldots, x_{N}, y\right)$ is uniformly finite on $X$.

So we can assume that $\operatorname{dim} X=N$, i.e., $X$ is open. Now we define:

$$
\begin{aligned}
& X_{1}=\left\{(\bar{a}, b) \in X: \bar{a} \text { is good for } \phi\left(x_{1}, \ldots, x_{N-1}, b, y\right)\right\} \\
& X_{2}=\left\{(\bar{a}, b) \in X: b \text { is good for } \phi\left(\bar{a}, x_{N}, y\right)\right\}
\end{aligned}
$$

(see Definition 3.4).

By $(3.5)_{N}$ there is a decomposition $P$ of $X$ which partitions both $X_{1}$ and $X_{2}$. 
Claim 5.8. Let $Y \in \mathcal{P}$ be open. Then $Y \subset X_{1}$ and $Y \subset X_{2}$.

Proof of Claim 5.8. As $Y$ is open we can find an open box $B \subset Y$. Let $(\bar{a}, b) \in B$ and let $\pi(B)$ be the projection of $B$ on the first $N-1$ coordinates. So $\pi(B)$ is an open cell in $M^{N-1}$. By $(3.7)_{N-1}, \phi\left(x_{1}, \ldots, x_{N-1}, b, y\right)$ is uniformly finite on $\pi(B)$. By (3.5) $)_{N-1}$ and (3.6) $)_{N-1}$ we can find an open cell $C \subset \pi(B)$ such that for some $k<\omega$, for all $\bar{a}^{\prime} \in C,\left|\phi\left(\bar{a}^{\prime}, b, y\right)^{M}\right|=k$ and moreover such that the first, second, $\ldots, k$ th point functions (if any) of $\phi(\bar{x}, b, y)$ are continuous on $C$. Let $\bar{a}^{\prime} \in C$. It is easy to see that $\bar{a}^{\prime}$ is good for $\phi(\bar{x}, b, y)$. So $\left(\bar{a}^{\prime}, b\right) \in X_{1}$. As $Y \cap X_{1}=\varnothing$ or $Y \subset X_{1}$ it follows that $Y \subset X_{1}$.

A similar argument shows that $Y \subset X_{2}$.

Now, by Claim 5.8, in order to complete the proof of $(3.7)_{N}(\mathrm{a})$ it is clearly enough to prove

LEMMA 5.9. Let $Y$ be an open cell in $M^{N}$ with the formula $\phi\left(x_{1}, \ldots, x_{N}, y\right)$ finite on $Y$ and such that for all $(\bar{a}, b) \in Y, \bar{a}$ is good for $\phi\left(x_{1}, \ldots, x_{N}, b, y\right)$ and $b$ is good for $\phi\left(\bar{a}, x_{N}, y\right)$. Then for all $\bar{c}_{1}, \bar{c}_{2}$ in $Y,\left|\phi\left(\bar{c}_{1}, y\right)^{M}\right|=\left|\phi\left(\bar{c}_{2}, y\right)^{M}\right|$.

ProOF OF LEMMA 5.9. Note that if the conclusion of the lemma fails, then for some $k<\omega, Y_{1}=\left\{\bar{c} \in Y:\left|\phi(\bar{c}, y)^{M}\right|=k\right\}$ is a nonempty proper definable subset of $Y$, and so by Proposition 2.4 and Lemma 2.3, $Y$ contains some boundary point $\bar{c}$ of $Y_{1}$ in $Y$. So, letting $B$ be an open box containing $\bar{c}$ and contained in $Y$, we see that $B$ contains points in $Y_{1}$ and also not in $Y_{1}$.

Thus it suffices to prove:

Let $B$ be an open box in $Y$. Then for all $\bar{c}_{1}, \bar{c}_{2}$ in $B$

$$
\left|\phi\left(\bar{c}_{1}, y\right)^{M}\right|=\left|\phi\left(\bar{c}_{2}, y\right)^{M}\right| \text {. }
$$

So let $\left(\bar{a}_{1}, b_{1}\right),\left(\bar{a}_{2}, b_{2}\right)$ be in $B$. By assumption, every $\bar{a} \in \pi(B)$ is good for $\phi\left(\bar{x}, b_{1}, y\right)$ (as for every $\bar{a} \in \pi(B),\left(\bar{a}, b_{1}\right) \in Y$ ).

Thus by $(3.7)_{N-1}(\mathrm{~b}),\left|\phi\left(\bar{a}_{1}, b_{1}, y\right)^{M}\right|=\left|\phi\left(\bar{a}_{2}, b_{1}, y\right)^{M}\right|$.

Similarly, by (3.7)(b) for $N=1$,

$$
\left|\phi\left(\bar{a}_{2}, b_{1}, y\right)^{M}\right|=\left|\phi\left(\bar{a}_{2}, b_{2}, y\right)^{M}\right| .
$$

Thus $\left|\phi\left(\bar{a}_{1}, b_{1}, y\right)^{M}\right|=\left|\phi\left(\bar{a}_{2}, b_{2}, y\right)^{M}\right|$.

This proves $(*)$, Lemma 5.9 and so also $(3.7)_{N}(\mathrm{a})$.

To complete the proof of Proposition 5.7 we need to prove $(3.7)_{N}(\mathrm{~b})$. But this is an immediate consequence of Lemma 5.9, using the fact that if $X$ is an open cell in $M^{N}$ and every $\bar{c} \in X$ is good for $\phi\left(x_{1}, \ldots, x_{N}, y\right)$, then for every $(\bar{a}, b) \in X, \bar{a}$ is good for $\phi\left(x_{1}, \ldots, x_{N-1}, b, y\right)$ and $b$ is good for $\phi\left(\bar{a}, x_{N}, y\right)$.

6. Conclusion. The results of $\S 4$, together with Propositions 5.1, 5.3 and 5.7, prove statements $(3.5)_{n},(3.6)_{n}$ and $(3.7)_{n}$ for all $n<\omega$. We now point out how Theorems $0.1-03$ follow.

Let $X \subset M^{N}$ be definable. By $(3.5)_{n}$, viewing $M^{n}$ as a cell, we obtain a decomposition $P$ of $M^{n}$ which partitions $X$. Then $X=\bigcup\{Y: Y \in P, Y \subset X\}$ and by Proposition 2.4, each $Y \in P$ is definably connected. This proves Theorem 0.1 .

Now for Theorem 0.2. Let $\phi(\bar{x}, y)$ be an $L$-formula $(l(\bar{x})=n)$. So for any $\bar{a} \in M^{n}, \phi(\bar{a}, y)^{M}$ is a finite union of intervals and points (as we assume $M$ is 0 -minimal). Let $\psi(\bar{x}, z)$ "say": $z$ is a boundary point of the set $\{y: \phi(\bar{x}, y)\}$. Thus 
$\psi(\bar{x}, z)$ is finite on $M^{n}$. By $(3.7)_{n}(\mathrm{a}), \psi(\bar{x}, z)$ is uniformly finite on $M^{n}$. Thus for some $k<\omega$, we have

$$
M \vDash(\forall \bar{x})(\{y: \phi(\bar{x}, y)\} \text { has at most } k \text { boundary points }) .
$$

So for any $N \equiv M$ and $\bar{a} \in N, \phi(\bar{a}, y)^{N}$ is a finite union of points and intervals. As $\phi$ was an arbitrary $L$-formula, this shows that $M$ is strongly 0 -minimal, proving Theorem 0.2.

Now for Theorem 0.3. Let $T=\operatorname{Th}(M)$, where $M$ is 0 -minimal. Thus, by Theorem 0.2, Theorem 0.1 holds for any $N \vDash T$. Let $\phi\left(x_{1}, \ldots, x_{n}, \bar{y}\right)$ be any $L$ formula. So for any $\bar{a}$ in a model $N$ of $T, \phi(\bar{x}, \bar{a})^{N}$ is a finite (disjoint) union of cells in $N^{n}$.

Now from the definition of a cell, it follows that for each $\bar{a}$ in a model $N$ of $T$, there is a formula $\psi(\bar{y})$ (without parameters) such that $\psi(\bar{a})$ expresses the fact that $\phi(\bar{x}, \bar{a})^{N}$ is a particular finite union of cells in $N^{n}$.

Let $\Psi(\bar{y})$ be the set of all possible such formulas $\psi(\bar{y})$, as $\bar{a}$ ranges over models of $T$. Thus $T \vDash(\forall \bar{y}) \vee \Psi(\bar{y})$.

By compactness, $T \vDash(\forall \bar{y}) \vee \Psi^{\prime}(\bar{y})$, where $\Psi^{\prime}(\bar{y})$ is some finite subset of $\Psi$. It clearly follows that for some $k<\omega$, for any $\bar{a}$ in $M$ (in fact in any model of $T), \phi(\bar{x}, \bar{a})^{M}$ is a finite disjoint union of at most $k$ cells in $M^{n}$. Each cell is definably connected by 2.4 . So clearly $\phi(\bar{x}, \bar{a})^{M}$ has at most $k$ definably connected components.

This proves Theorem $0.3(\mathrm{a})$. Now if the underlying order of $M$ is $(\mathbf{R},<)$, then it is easy to see that any cell in $M^{n}$ is actually connected. Thus $\phi(\bar{x}, \bar{a})^{M}$ has at most $k$ connected components, as $\bar{a}$ ranges ove $\mathrm{r}^{m}(\bar{m}=l(\bar{y}))$, proving Theorem $(0.3)(b)$.

\section{REFERENCES}

[B] G. W. Brumfiel, Partially ordered rings and semi-algebraic geometry, London Math. Soc. Lecture Note Series no. 37, Cambridge Univ. Press, London, 1979.

[C] G. E. Collins, Quantifier elimination for real closed fields by cylindrical algebraic decomposition, Automata Theory and Formal Languages, Springer-Verlag, Berlin and New York, 1975.

[D] L. van den Dries, Remarks on Tarski's problem concerning $(\mathbf{R},+, \cdot, \exp )$, Logic Colloquium '82 (Lolli, Longo and Marcja, eds.), North-Holland, Amsterdam, 1984.

[M] J. Mather, Stratifications and mappings, Dynamical Systems (M. Peixoto, ed.), Academic Press, New York, 1973.

[PS1] A. Pillay and C. I. Steinhorn, Definable sets in ordered structures, Bull. Amer. Math. Soc. (N.S.) 11 (1984), 159-162.

[PS2] _ Definable sets in ordered structures. I, preprint, 1984; Trans. Amer. Math. Soc. 295 (1986), 565-592.

[T] A. Tarski, A decision method for elementary algebra and geometry, 2nd rev. ed., Univ. of California Press, Berkeley and Los Angeles, 1951.

[W] H. Whitney, Elementary structure of real algebraic varieties, Ann. of Math. (2) 66 (1957), $545-556$.

Department of MATHEMATICS, University of Notre DAmE, Notre DAme, INDIANA 46556 (Current address of J. F. Knight and A. Pillay)

Department of Mathematics, Vassar College, Poughkeepsie, New York 12601 (Current address of C. Steinhorn) 